\title{
Multiple Directional Differentiation Difference of Neonatal Rat Fibroblasts from Six Organs
}

\author{
Yuqiao Chang ${ }^{a, b}$ Kang Guoc Qiong Li ${ }^{b}$ Cixia Li ${ }^{b}$ Zhikun Guo ${ }^{b}$ He Lia
}

aDepartment of Human Anatomy and Embryology, Tongji Medical College, Huazhong University of

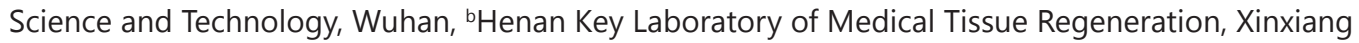
Medical University, Xinxiang, 'Tumor Department, Third Affiliated Hospital, Xinxiang Medical University, Xinxiang, P.R. China

\section{Key Words}

Fibroblasts - Immunofluorescence stainning - Stem cell markers • Heterogeneity • Directed differentiation

\begin{abstract}
Background/Aims: Fibroblasts are abundantly distributed throughout connective tissues in the body and are very important in maintaining the structural and functional integrity. Recent reports have proved that fibroblasts and mesenchymal stem cells share much more in common than previously recognized. The aim of this study was to investigate comparative studies in fibroblasts on the differences in the expression of molecular markers and differentiation capacity from different organs. Methods: Combined trypsin/collagenase enzymes digestion method was used to isolate and culture the fibroblasts derived from heart, liver, spleen, lung, kidney and skin. Cell activity was determined by methyl thiazolyl tetrazolium (MTT) assay. Common molecular markers for fibroblasts such as vimentin, DDR2 and FSP1, stem cell markers nanog, c-kit and sca-1 were detected by RT-PCR, immunofluorescence and western blotting. The osteogenic, adipogenic and cardiogenic differentiations of fibroblasts were performed by inductive culture in special mediums, and analyzed by Alizarin red, Oil red $\mathrm{O}$ and immunofluorescence staining of CTnT respectively. Results: The proliferation rate of fibroblasts in lung was faster than in other five organs. Common molecular markers for fibroblasts were expressed differently in different organs. DDR2 was strongly expressed in fibroblasts in the heart, partly expressed in the heart, skin, liver and spleen. Interestingly, no expression of DDR2 was detected in liver and kidney. However, vimentin and FSP1 were consistently expressed in fibroblasts from skin, liver, kidney, spleen and lung. nanog expression in fibroblasts from lung was less than that from heart, skin, liver and spleen $(P<0.01)$. c-kit expression in fibroblasts from heart, skin and kidney was higher than that from spleen $(P<0.05)$, while the c-kit positive fibroblasts from liver was obviously higher than that from spleen $(P<0.01)$. But sca1 expression in fibroblasts from lung was the lowest among six organs $(P<0.01)$. Directed differentiation in vitro had demonstrated that skin fibroblasts had the strongest multiple differentiation potential, and the next was cardiac fibroblasts. And fibroblasts in liver and kidney had the advantage in myocardial differentiation, while fibroblasts in spleen only had

Zhikun Guo, MD, PhD

and $\mathrm{He} \mathrm{Li}, \mathrm{MD}, \mathrm{PhD}$

Henan Key Laboratory of Medical Tissue Regeneration, Xinxiang Medical University Xinxiang 453003, (P.R. China); Department of Human Anatomy and Embryology, Tongji Medical College, Huazhong University of Science and Technology, Wuhan 430030, (P.R. China); E-Mail gzk@xxmu.edu.cn / heli@mails.tjmu.edu.cn
\end{abstract}

KARGER 


\section{Cellular Physiology Cell Physiol Biochem 2016;39:157-171

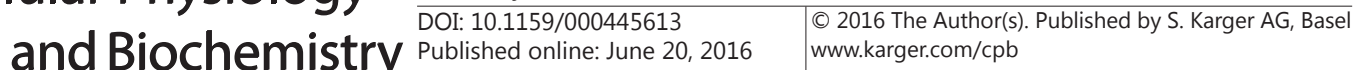 \\ Chang et al.: Heterogeneity in Fibroblasts from Different Organs}

the advantage in osteogenic differentiation. Conclusions: There are obvious heterogeneity in molecular markers and muti-directional differentiation in fibroblasts from six organs.

(C) 2016 The Author(s)

Published by S. Karger AG, Basel

\section{Introduction}

Fibroblasts are abundantly distributed throughout connective tissues in the body and are very important in maintaining the structural and functional integrity. Fibroblasts have traditionally been viewed as a uniform cell type with supporting and secreting function regardless of the origins of tissue. Recent studies have proved that there are many subpopulations in fibroblasts, which have significant heterogeneity in cell morphology $[1,2]$, growth characteristics [3], collagen synthesis [4], cytokines and surface receptors $[5,6]$. More and more scientists pay close attention to their function in organism because fibroblasts and mesenchymal stem cells share much more in common than previously recognized [7-9]. Indeed, lung fibroblasts have been shown to be heterogeneous in cell surface marker expression, as well as in their levels of collagen production [10]. Moreover, periodontal fibroblasts also show heterogeneity based on morphology, glycogen pools and collagen production [10]. Additionally, fibroblasts from different anatomic sites have distinct transcriptional patterns [11].

However, there is lack of comparative studies on fibroblasts on the differences in the expression of molecular markers and differentiation capacity from different organs. Combined enzyme digestion method was used to isolate and culture fibroblasts derived from heart, liver, spleen, lung, kidney and skin in order to explore the differences of biological phenotypes. Common molecular markers for fibroblasts such as vimentin, FSP1 and DDR2, stem cell markers Nanog, c-kit and sca-1 were detected to identify their heterogeneity. Adipocytic, osteogenic, neural and cardiac induction assays in fibroblasts were also done in order to select optimal organs, to provide the reliable experimental support and optimal seed cells in situ organ repair.

\section{Material and Methods}

\section{Animals}

Neonatal Sprague-Dawley rats (1-3 day-old, $7.1 \pm 0.31 \mathrm{~g}, \mathrm{n}=60)$ were utilized in this study. Animal treatment was performed according to the guidelines of The Ministry of Science and Technology of the People's Republic of China [(2006)398] and approved by the Xinxiang Medical University Animal Care Committee (No 030032).

\section{Isolation and culture of fibroblasts}

Neonatal rats were weighed and executed then the heart, lung, liver, spleen, kidney and skin were obtained and washed separately with PBS in aseptic conditions. The tissues from heart, lung, liver, spleen, kidney and skin were minced into small pieces of about $1 \mathrm{~mm}^{3}$ and incubated with $0.25 \%$ trypsin and $0.125 \%$ collagenase enzymes (Sigma-Aldrich, St. Louis, MO, USA) and the digestion time was $40 \mathrm{~min}, 30$ min, $25 \mathrm{~min}, 20 \mathrm{~min}, 35 \mathrm{~min}$ and $80 \mathrm{~min}$ respectively. The suspension was centrifuged at $1200 \mathrm{r} / \mathrm{min}$ for 5 min. The cellular pellet was filtered through a $40 \mu \mathrm{m}$ cell strainer (BD Biosciences, San Jose, CA) and was re-suspended in DMEM-LG culture medium supplemented with $8 \%$ fetal calf serum, $100 \mathrm{U} / \mathrm{ml}$ penicillin and $100 \mathrm{mg} / \mathrm{ml}$ streptomycin (Sigma-Aldrich). The cells were plated in $25 \mathrm{~cm}^{2}$ dishes and cultured in a $\mathrm{CO}_{2}$ incubator at $37^{\circ} \mathrm{C}$. After $40 \mathrm{~min}$, suspensions were discarded and complete culture media were replenished. Then the culture media were changed every $48 \mathrm{~h}$, when cells reached $85 \%$ confluence, and the cells were harvested for further passage following $0.25 \%$ trypsin/0.01\% EDTA digestion (Amresco, Solon, Ohio, USA). The fibroblasts of passage 3 were plated in 24 -well plates and cultured in a $\mathrm{CO}_{2}$ incubator at $37^{\circ} \mathrm{C}$. When cells reached $70 \%$ confluence then fixed with $4 \%$ paraformaldehyde. 


\section{Cellular Physiology Cell Physiol Biochem 2016;39:157-171 \begin{tabular}{l|l|} 
and BOI: 10.1159/000445613 2016 & $\begin{array}{l}\text { C) 2016 The Author(s). Published by S. Karger AG, Basel } \\
\text { www.karger.com/cpb }\end{array}$
\end{tabular} \\ Chang et al.: Heterogeneity in Fibroblasts from Different Organs}

Identification of fibroblasts by immunofluorescence staining

Isolated fibroblasts from six organs were fixed in $4 \%$ paraformaldehyde for 30 min at room temperature, subsequently washed with PBS and treated with $0.3 \%$ Triton X-100(Sigma) in PBS for 10 min. Then the samples were incubated with PBS containing $2 \%$ bovine serum albumin (BSA) at $37^{\circ}$ for $10 \mathrm{~min}$. Incubation with the primary antibodies (Rabbit anti-vimentin, Abcam, 1:100; Rabbit anti-FSP1, Abcam, 1:200; Goat anti-DDR2, Santa Cruz, 1:300) was performed at $4^{\circ}$ overnight. The samples were subsequently incubated FITC- or Cy3-conjugated secondary antibodies (Beyotime, 1:500). The nuclei were counterstained with 5 mg /L DAPI (Roche). Negative controls were obtained by following all the same protocol but the primary antibodies. All experiments were performed in triplicate.

\section{Cell growth curve from different organs by MTT assay}

Primarily cultured fibroblasts from heart, lung, liver, spleen, kidney and skin were seeded into 8,000/ well in a $\mathrm{CO}_{2}$ incubator at $37^{\circ} \mathrm{C}$. MTT assay were detected by $5 \mathrm{mg} / \mathrm{ml}$ MTT dissolved in PBS, $3 \mathrm{~h}$ before the end of the incubation, add $20 \mu \mathrm{l}$ of solution, then incubate the plate at $37^{\circ} \mathrm{C}$ for $2 \mathrm{~h}$ and add $150 \mu \mathrm{l}$ DMSO to each well and pipette up and down to dissolve crytals. Then transfer to plate reader and measure absorbance at wavelength $570 \mathrm{~nm}$. All experiments were performed in triplicate. The cell growth curve was determined by the optical density (OD) value.

RT-PCR of molecular markers of fibroblasts from six organs

Total RNA was extracted from passage 3 fibroblasts isolated from six organs. Reverse transcription and PCR were done referring to TaKaRa RNA PCR kit. Primers for PCR amplification were synthesized by Shanghai Sangon Biological Engineering and Technology Service Co. Ltd. Sequences of the PCR primers were seen in Table 1. PCR reaction mixture was subjected to pre-denaturation at $94^{\circ} \mathrm{C}$ for 5 min followed by 30 amplification cycles each consisting of denaturation at $94^{\circ} \mathrm{C}$ for $15 \mathrm{~s}$, annealing at $55^{\circ} \mathrm{C}$ for $40 \mathrm{~s}$ and extension at $72^{\circ} \mathrm{C}$ for $30 \mathrm{~s}$, and then a final elongation cycle at $72^{\circ} \mathrm{C}$ for $10 \mathrm{~min}$ was performed.

\section{Western blotting assay}

Fibroblasts of passage 3 from heart, lung, liver, spleen, kidney and skin were lysed and protein was extracted. The number of the cell was about $1 \times 10^{8}$. The protein concentration was measured by BCA assay and was subjected to pre-denaturation at $100^{\circ} \mathrm{C}$ for $5 \mathrm{~min} .100 \mu \mathrm{g}$ of total protein were loaded in each

Table 1. Sequences of the PCR primers

\begin{tabular}{lll}
\hline gene & Forward primer $\left(5^{\prime} \rightarrow 3^{\prime}\right)$ & Reverse primer $\left(5^{\prime} \rightarrow 3^{\prime}\right)$ \\
\hline nanog & AGAAGATGCGGACTGTGTTC & GCTCAGGTTCAGAATGGTAGA \\
sca-1 & CTAGCCAGAGACATCAGGA & CCATAGGACCAGACATCAC \\
vimentin & GCTACTATGATGTCCTGTGT & AACAACTTCTGCCACCTG \\
DDR2 & CAATAACAACCACTCCTCATC & AACTCTTCCACAGCCACATC \\
FSP1 & TCAGCACTTCCTCTCTCTTGG & CACCCTCGTTGCCTGAGTAT \\
GADPH & TCCCTCAAGATTGTCAGCAA & AGATCCACAACGGATACATT \\
\hline
\end{tabular}

Table 2. Source and drops of grouped antibodies for immunofluorescence staining

\begin{tabular}{cl}
\hline \multicolumn{1}{c}{ Primary antibodies } & Secondary antibodies \\
\hline (1) Mouse anti-vimentin $(1: 100)$ & Goat anti-mouse IgM-FITC (1:500) \\
Rabbit anti-sca-1 (1:200) & Goat anti-rabbit IgM-Cy3 (1:500) \\
(2) Mouse anti-vimentin (1:100) & Goat anti-mouse IgM-FITC (1:500) \\
Rabbit anti-c-kit (1:200) & Goat anti-rabbit IgM-Cy3 (1:500) \\
(3) Mouse anti-vimentin (1:100) & Goat anti-mouse IgM-FITC (1:500) \\
Rabbit anti-nanog (1:300) & Goat anti-rabbit IgM-Cy3 (1:500) \\
\hline
\end{tabular}




\section{Cellular Physiology Cell Physiol Biochem 2016;39:157-171 \begin{tabular}{l|l} 
DOI: 10.1159/000445613 & $\begin{array}{l}\text { O 2016 The Author(s). Published by S. Karger AG, Basel } \\
\text { www.karger.com/cpb }\end{array}$
\end{tabular} \\ Chang et al.: Heterogeneity in Fibroblasts from Different Organs}

well on 8-10\% SDS-PAGE gels and electrophoretically blotted onto a PVDF membrane at constant voltage of $90 \mathrm{~V}$ for $90 \mathrm{~min}$. The membrane was blocked for $1 \mathrm{~h}$ with $5 \%$ nonfat dry milk at room temperature. Primary antibodies were added respectively like FSP1 (Abcam, 1:400), CD73 (BD, 1:1000), sca-1 (Merk, 1:500), c-kit (Santa Cruz, 1:1000), vimentin (Abcam, 1:1000), Nanog (Santa Cruz, 1:500) overnight at $4^{\circ} \mathrm{C}$, then the PVDF membrane was washed 3 times with TBST for 10 min each. Incubate the membrane with the HRP-conjugated secondary antibody diluted to 1:2000 in blocking solution at room temperature for $1 \mathrm{~h}$. Wash membrane 3 times with TBST for 10 min each. Prepare ECL substrate according to the manufacture's instructions. Incubate the membrane with substrate completely for 1-5min. Exposure autoradiography film exposure under a chemiluminescence imaging system. The results were analyzed with Image-Pro Plus 6.0 software.

\section{Immunofluorescence stainning for cell surface markers}

Fibroblasts from six organs grown on coverslips were fixed with 4\% paraformaldehyde for $20 \mathrm{~min}$, subsequently washed with PBS three times, and treated with $0.3 \%$ Triton X-100 in PBS for 10 min at room temperature. The samples were then blocked with $2 \%$ BSA for $20 \mathrm{~min}$, and incubated with the mixed primary antibodies grouped as Table 2 at $4^{\circ} \mathrm{C}$ overnight (Table 2). The samples were subsequently incubated with FITC- or Cy3-conjugated secondary antibodies and DAPI (Table 2). Control staining was performed without primary antibody. All experiments were performed in triplicates.

\section{Directional differentiation culture}

Fibroblasts from six organs at passage 3 were plated at $1 \times 10^{5}$ cells/well in 24 -well plates in DMEM supplemented with 8\% FBS and were cultured for $4 \mathrm{~h}$. For cardiomyogenic differentiation, cells were treated with complete DMEM supplemented with $10 \mu \mathrm{M}$ 5-azacytidine (5-Aza; Sigma, St. Louis, MO, USA) for 24 h. Next, cells were washed twice with PBS, the medium was replaced by complete medium without 5-Aza. The medium was changed every two days. Cells were fixed on day 30 and cardiomyogenic differentiation was identified by immunostaining to measure expression of myocardial cell marker cTnT. For adipogenic, osteogenic and neural differentiation induction, the culture medium was replaced with low-glucose DMEM containing 10\% FBS plus adipogenic reagents $(1 \mu \mathrm{M}$ Dexamethasone, $0.01 \mathrm{mg} / \mathrm{ml}$ insulin, $200 \mu \mathrm{M}$ indometacin, $0.5 \mathrm{mM}$ Isobutyl- methylxanthine), $10 \% \mathrm{FBS}$ plus osteogenic reagents $(0.1 \mu \mathrm{M}$ Dexamethasone, $10 \mathrm{mM}$ sodium $\beta$-glycerophosphate, $0.05 \mathrm{mM}$ L-Ascorbic Acid) and DMEM/F-12 medium plus neural reagents $(0.2 \mu \mathrm{g} / \mathrm{L}$ basic fibroblast growth factor, $0.2 \mu \mathrm{g} / \mathrm{L}$ epidermal growth factor, $2 \%$ B27) respectively. The adipogenic or osteogenic media was changed every 2 days. Control group was maintained using lowglucose DMEM containing 10\% FBS. Adipogenic differentiation was identified by Oil Red 0 following 14 days of adipogenic induction, osteogenic differentiation was identified by Alizarin Red following 21 days of osteogenic induction and neural differentiation was identified by immunofluorescence staining for neuronspecific enolase (NSE). The images were captured by microscopes and 5 fields were randomly selected from each well in 24-well plates, finally 30 fields were chosen to get the differentiation rate which was N1/ $\mathrm{N} \times 100 \%$, while $\mathrm{N} 1$ was the number of the differentiation cells and $\mathrm{N}$ was the total number of viable cells evaluated under the same vision.

\section{Statistical analysis}

The data were analyzed by SPSS 17.0 software. The rate of co-expression equated the total number of cells with nuclei was divided by the number of co-expression in 30 random fields. The results were shown by mean \pm standard deviation $(\bar{X} \pm S$ ), and two different samples were compared by T-test, while multiple samples were compared with single-factor analysis of variance according to the $\alpha=0.05, P<0.05$ was considered as a statistically significant difference.

\section{Results}

The morphology of isolated fibroblasts from six organs

Neonatal rat fibroblasts from heart, lung, liver, spleen, kidney and skin were isolated and began to adhere to the wall in 30-40 min. They showed triangle, shuttle or irregular polygons and had a branched cytoplasm surrounding an elliptical, speckled nucleus having 


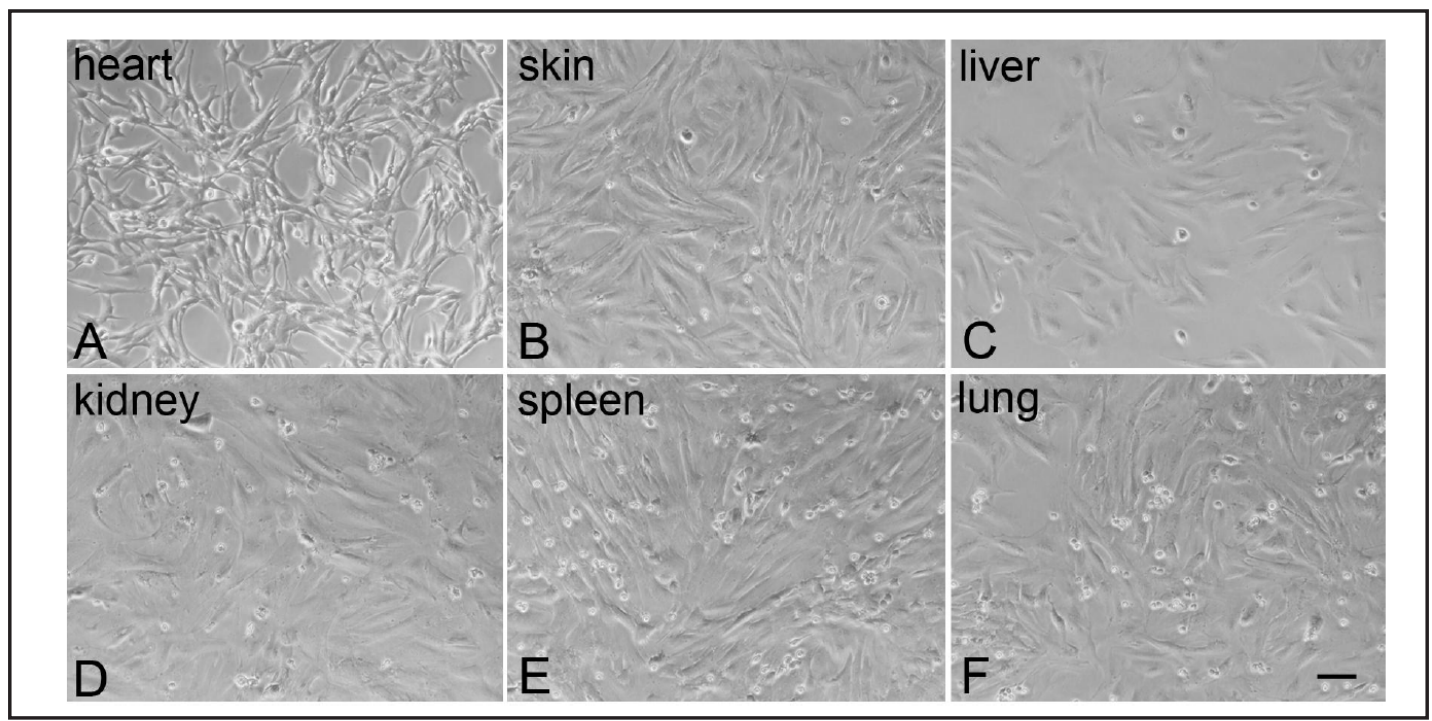

Fig. 1. Morphology of cultured fibroblasts passage 3 from six organs under phase-contrast microscope. (A) cardiac fibroblasts on $3 \mathrm{~d}$. (B) skin fibroblasts on $2 \mathrm{~d}$. (C) fibroblasts in liver on $3 \mathrm{~d}$. (D) fibroblasts in kidney on $4 \mathrm{~d}$. (E) splenic fibroblasts on $4 \mathrm{~d}$. (F) fibroblasts in lung on $2 \mathrm{~d}$. Scale bar $=50 \mu \mathrm{m}$.

Fig. 2. Vimentin, $D D R 2$ and $F S P 1$ in fibroblasts from six organs were detected by RT-PCR. Vimentin and FSP1 were detected in fibroblasts from six organs, while DDR2 expressed in heart, spleen, liver and spleen, but not in kidney and lung.

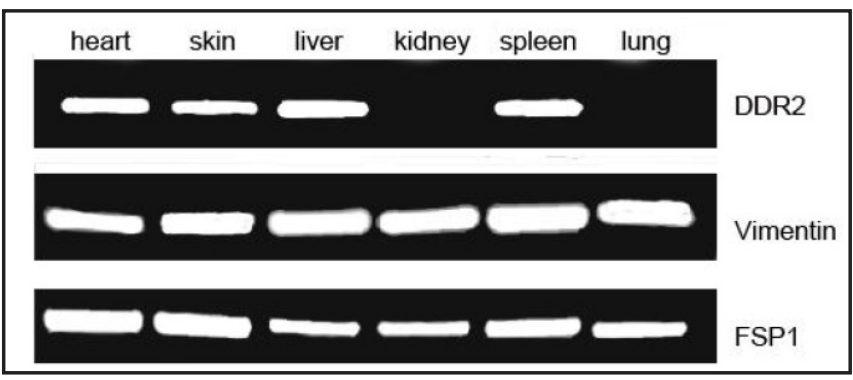

two or more nucleoli. They could not beat spontaneously and had high proliferation and metabolic activity. Isolated fibroblasts from different organs had different morphology in cell shape and volume (Fig. 1). Fibroblasts in heart showed triangle, shuttle shaped and polygons and more than $40 \%$ with double nuclei. Proliferated phases were visible obviously (Fig. 1A). Skin fibroblasts were the most diverse in morphology, except for shuttle shaped, triangle and polygons, they showed star-like or oval shapes and mutually interwove like a mesh (Fig. 1B). There were more triangle and shuttle shaped in liver (Fig. 1C), while more polygons and larger cell body in kidney (Fig. 1D). Splenic fibroblasts had smaller cell body and more shuttle shaped, and the percentage of double nuclei accounted for $24 \%$ (Fig. 1E), but most fibroblasts in lung were triangle (Fig. 1F).

\section{Difference gene expression in molecular markers from six organs}

Vimentin, discoidin domain receptor 2(DDR2) and Fibroblast-specific protein 1(FSP1) in fibroblasts from six organs were detected by RT-PCR. Vimentin and FSP1 were picked up in fibroblasts from six organs, while DDR2 was expressed in heart, spleen, liver and spleen, but not in kidney and lung (Fig. 2).

\section{Growth characteristics in fibroblasts from different organs}

There were also differences in cell growth curve and lag phase in fibroblasts from six organs. The lag phase was about 8-48 h in skin, lung and liver, 8-72 $\mathrm{h}$ in spleen, heart and kidney. The log phase was nearly $48-72 \mathrm{~h}$ in skin and lung, then to stationary phase after $72 \mathrm{~h}$, while the log phase was $72-120 \mathrm{~h}$ in spleen and kidney, and $96 \mathrm{~h}$ in heart. Isolated 


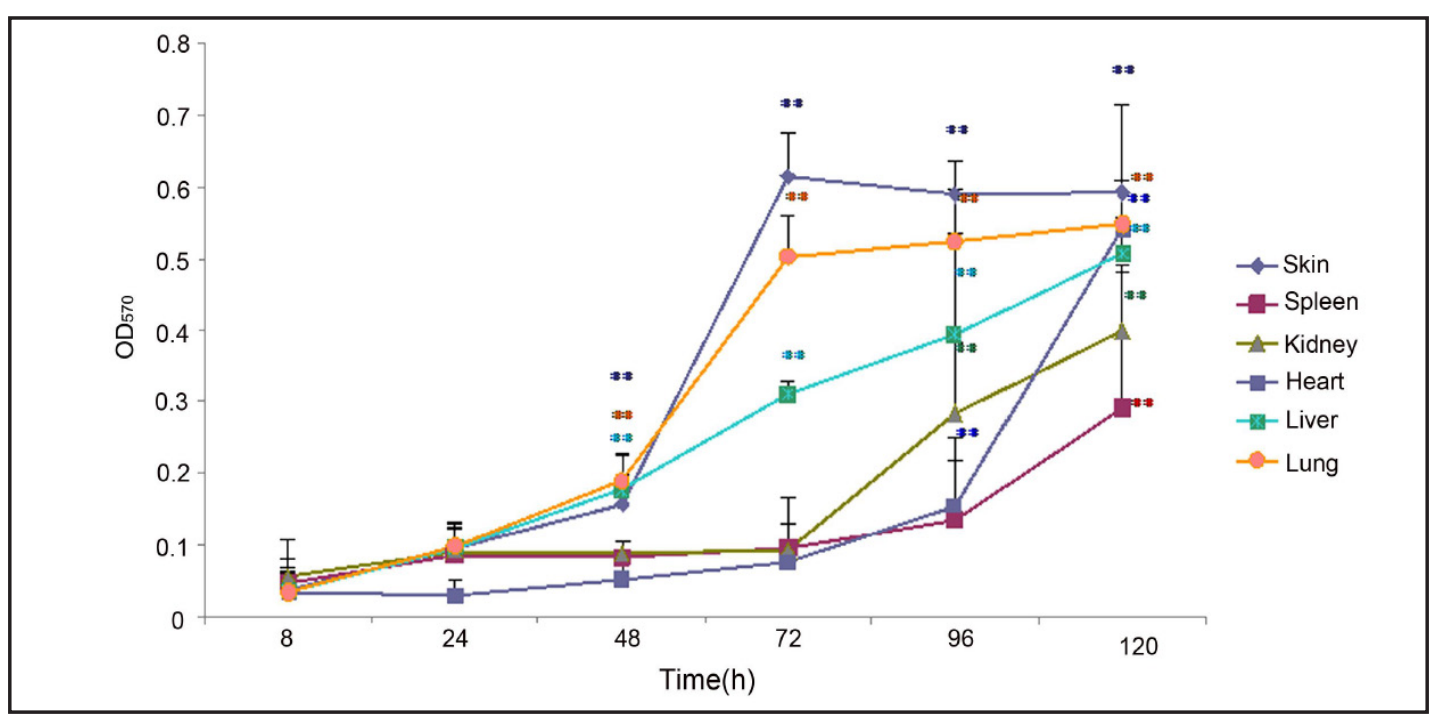

Fig. 3. Cell growth curve of fibroblasts from six organs. The OD value in skin, lung and liver had significantly increased after being cultured for $48 \mathrm{~h}$ than that for $8 \mathrm{~h}\left({ }^{* *} P<0.01\right)$. There was significantly increase in kidney and heart cultured at $96 \mathrm{~h}\left({ }^{* *} P<0.01\right)$. At $120 \mathrm{~h}$, the OD value in spleen had significantly increased $\left({ }^{* *} P<0.01\right)$.

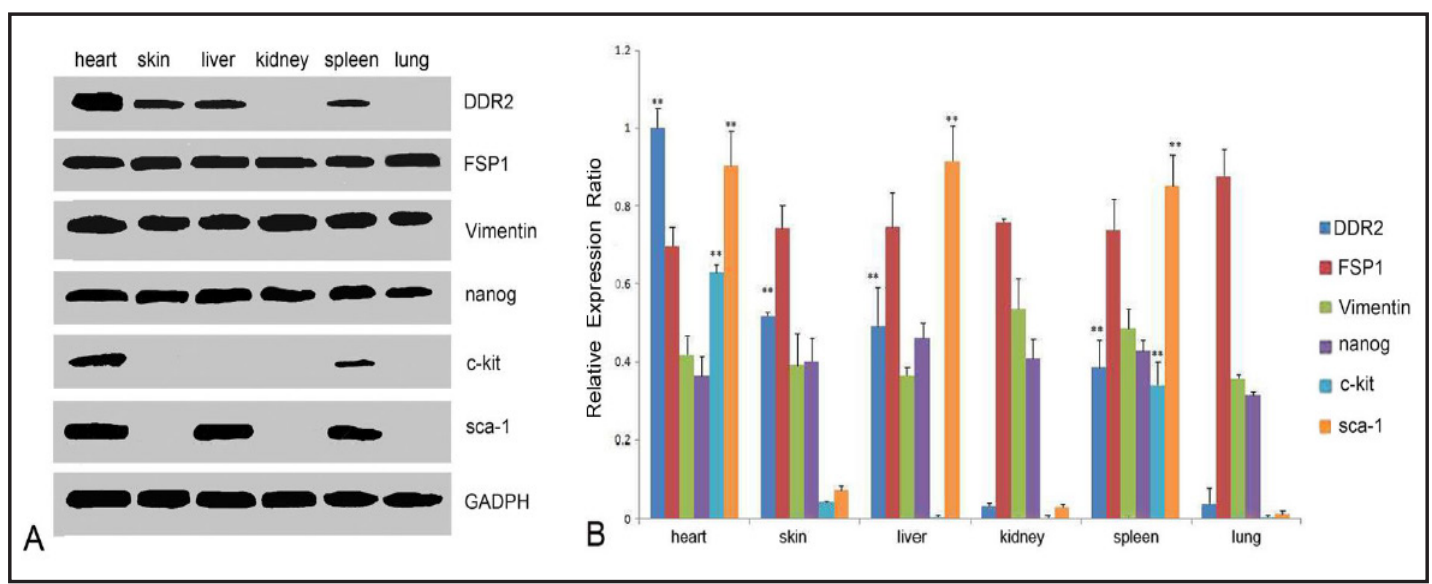

Fig. 4. Western blotting detection of DDR2, FSP1, vimentin, nanog, c-kit and sca-1 protein in fibroblasts from six organs. vimentin and FSP1 were all detected in fibroblasts from six organs and there were no significantly difference among six organs $(P>0.05)$. DDR2 was detected mainly in heart, partly in skin, liver and spleen, not in lung and kidney. sca-1 expression in fibroblast from heart, liver and spleen were more than that in fibroblasts from other three organs $\left({ }^{* *} P<0.01\right)$. C-kit expression in fibroblasts from heart was the most popular than other five organs $(* * P<0.01)$.

fibroblasts from six organs all showed a typical S-shaped curve from lag phase, log phase and stationary phase respectively.

Though comparison with proliferation rate in fibroblasts from skin, spleen, kidney, heart and liver in different periods, there were difference in Fig. 3. The OD value in skin, lung and liver had significantly increased after being cultured for $48 \mathrm{~h}$ than that for $8 \mathrm{~h}(P<0.01)$. The OD value in kidney and heart had significantly increased after being cultured for $96 \mathrm{~h}$ than that for $8 \mathrm{~h}(P<0.01)$. There was significantly difference in spleen cultured at $120 \mathrm{~h}(P<$ $0.01)$. The proliferation rate in fibroblasts at $72 \mathrm{~h}$ was skin $>$ lung $>$ liver $>$ kidney $=$ spleen $>$ heart, skin $>$ lung $>$ liver $>$ kidney $>$ heart $>$ spleen at $96 \mathrm{~h}$, and skin $>$ lung=heart $>$ liver $>$ kidney $>$ spleen at $120 \mathrm{~h}$. These results showed that the proliferation rate in skin was fasted, while the lag phase of fibroblasts in heart was longest up to almost $96 \mathrm{~h}$.

\section{KARGER}



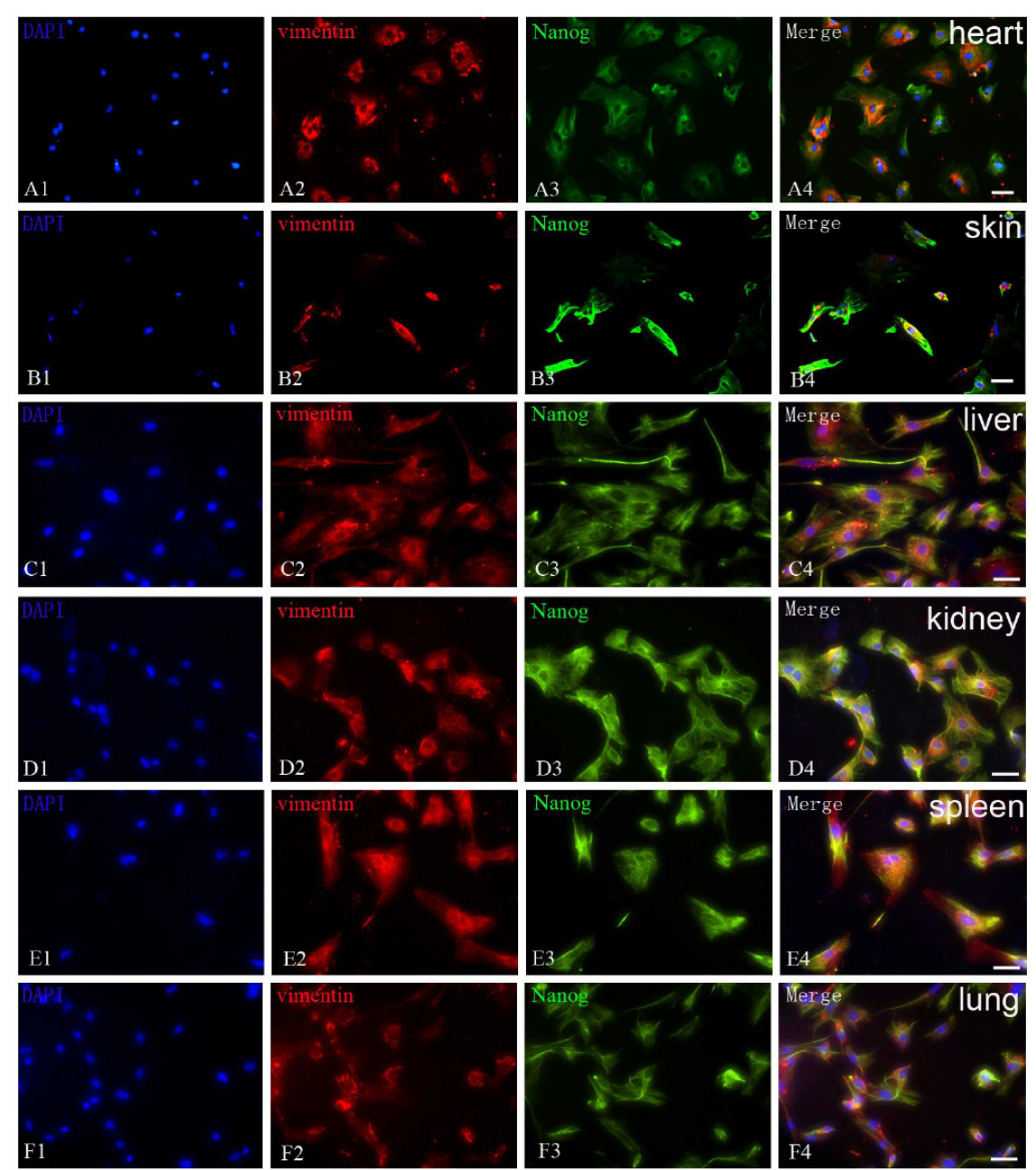

Fig. 5. The co-expression of vimentin and nanog protein in neonatal rats heart, liver, spleen, lung, kidney and skin by immunofluorescence staining. The nuclear stained by DAPI is shown in A1-F1; The red in A2-F2 and the green in A3-F3 were stained by vimentin marked by Cy3 and nanog protein marked by FITC; A4-F4 shown the merge result of the left three columns, the yellow indicate co-expression of vimentin and nanog protein. Scale bar $=50 \mu \mathrm{m}$.

\section{Comparison of protein expression in cultured fibroblasts from different organs}

Total proteins of fibroblasts from six organs were extracted and were detected by western blotting. Consistent with RT-PCR, there were also differences of protein expression in fibroblasts from different organs. Vimentin and FSP1 were all detected in fibroblasts from six organs and there were no significantly difference among six organs $(P>0.05)$. DDR2 was detected mainly in heart, partly in skin, liver and spleen, not in lung and kidney (Fig. 4A). There were no significantly difference in nanog expression in fibroblasts from six organs $(P$ $>0.05$, Fig. 4A), but sca-1 expression in fibroblast from heart, liver and spleen were more than that in fibroblasts from other three organs (Fig. 4B). C-kit expression in fibroblasts from heart was the most popular than other five organs $(P<0.01)$.

Differential expression of nanog, c-kit and sca-1 in fibroblasts from six organs by immunofluorescence staining

Isolated fibroblasts in vitro from six organs were detected by immunofluorescence double labeling. Vimentin was the major cytoskeletal component of fibroblasts, clearly visible in the distribution within the cell (Fig. 5-7). Nanog were mainly detected within 


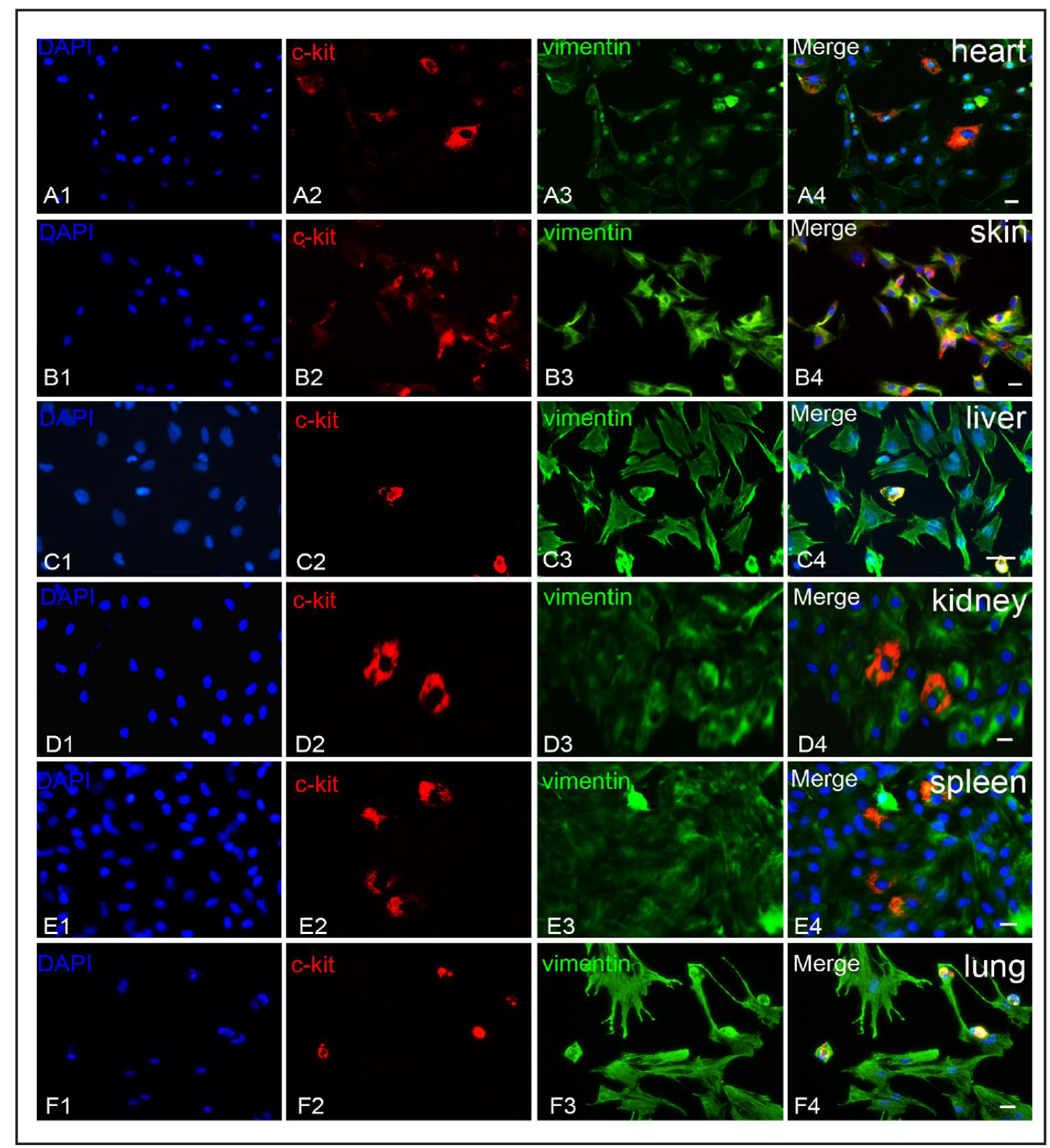

Fig. 6. The co-expression of vimentin and c-kit protein in neonatal rats heart, liver, spleen, lung, kidney and skin by immunofluorescence staining. The nuclear stained by DAPI is shown in A1-F1; the red in A2-F2 and the green in A3-F3 were stained by c-kit marked by Cy3 and vimentin marked by FITC; A4-F4 shown the merge result of the left three columns, the yellow indicate co-expression of vimentin and c-kit protein. Scale bar $=30 \mu \mathrm{m}$.

cytoplasm, partly in nuclei of fibroblasts from six organs (Fig. 5). C-kit was also detected in cytomembrane and cytoplasm, but majority of positive fibroblasts were in round and oval shape (Fig. 6). Sca-1 expressed in nuclei of fibroblasts from liver, kidney, spleen and lung, while sca-1 was detected not only in nuclei, but also in cytoplasm of fibroblasts from heart and skin (Fig. 7).

\section{Multipotential differentiation in fibroblasts from six organs}

Fibroblasts from six organs were induced by 5-aza respectively, then cells morphology had more changes in shape and proliferation comparing to the control group without 5-aza. The majority of cells showed triangle and spindle before induction. But they steadily turned KARGER 

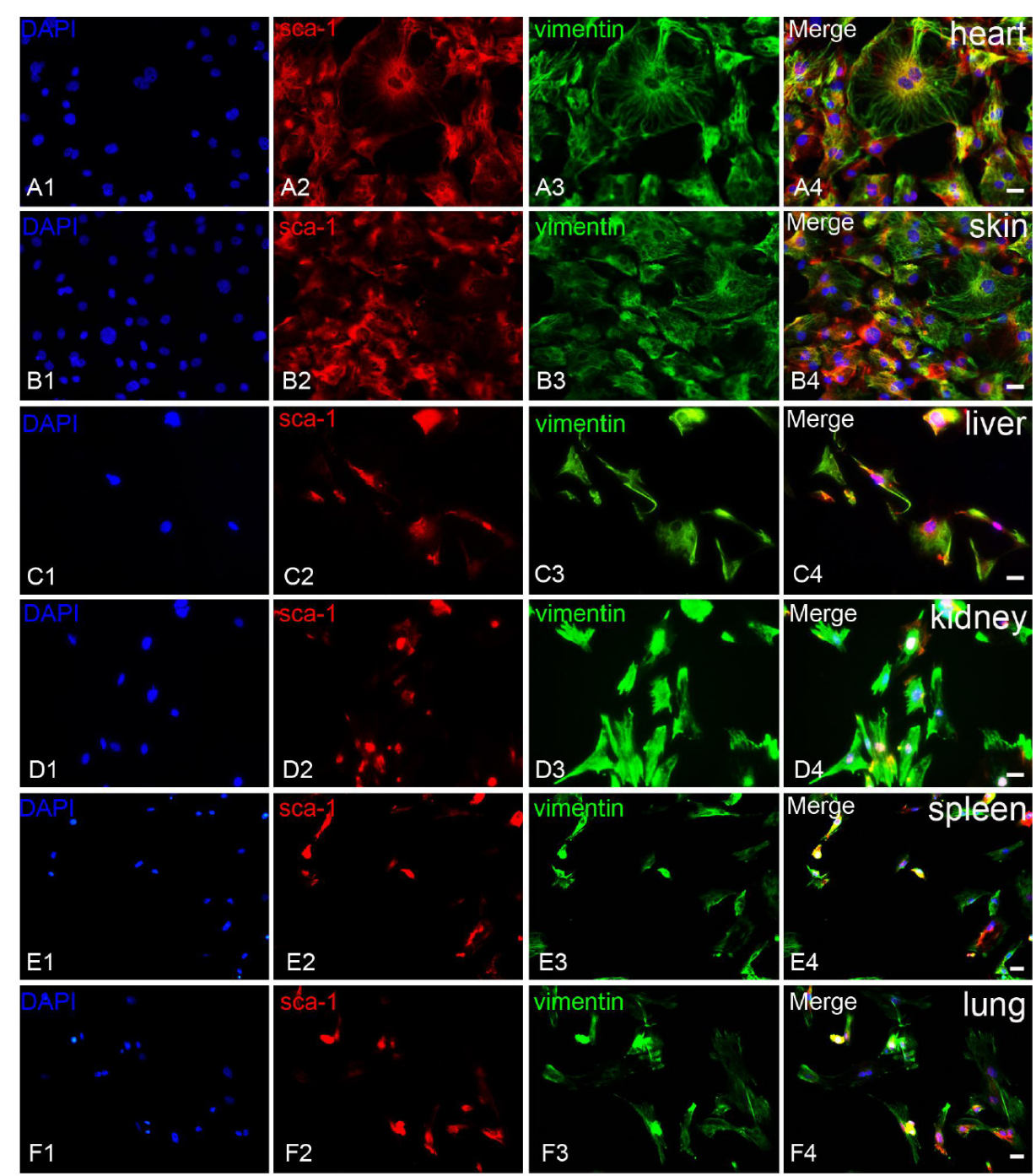

Fig. 7. The co-expression of vimentin and sca-1 protein in neonatal rats heart, liver, spleen, lung, kidney and skin by immunofluorescence staining. The nuclear stained by DAPI is shown in A1-F1; the red in A2-F2 and the green in A3-F3 were stained by sca-1 marked by Cy3 and vimentin marked by FITC; A4-F4 shown the merge result of the left three columns, the yellow indicate co-expression of vimentin and sca-1 protein. Scale bar $=30 \mu \mathrm{m}$.

to round or rod and slow proliferation after induction, and the induced cells were gradually bigger with increased secretions. The cells in control group were woven through the bottom of the dish (data not shown). After $28 \mathrm{~d}$ induction, some cells had positive expression for cTnT (Fig. 8A-D).

Fibroblasts were induced by neuronal induction medium and their shape turned to long rod from flat shape, their cell processes were gradually elongated and slow proliferation. At the same time, nuclei became smaller, cytoplasm shrinked and cell parameters significantly enhanced. After $5 \mathrm{~d}$, the cells grew into secondary branches and their long protrusions connected into a network between adjacent cells, but there were still a few fibroblasts whose morphology did not change obviously. NSE protein was detected strongly in induced cells on 7th day (Fig. 8E-H), which showed that fibroblasts could be differentiated into neuron-like cells. 


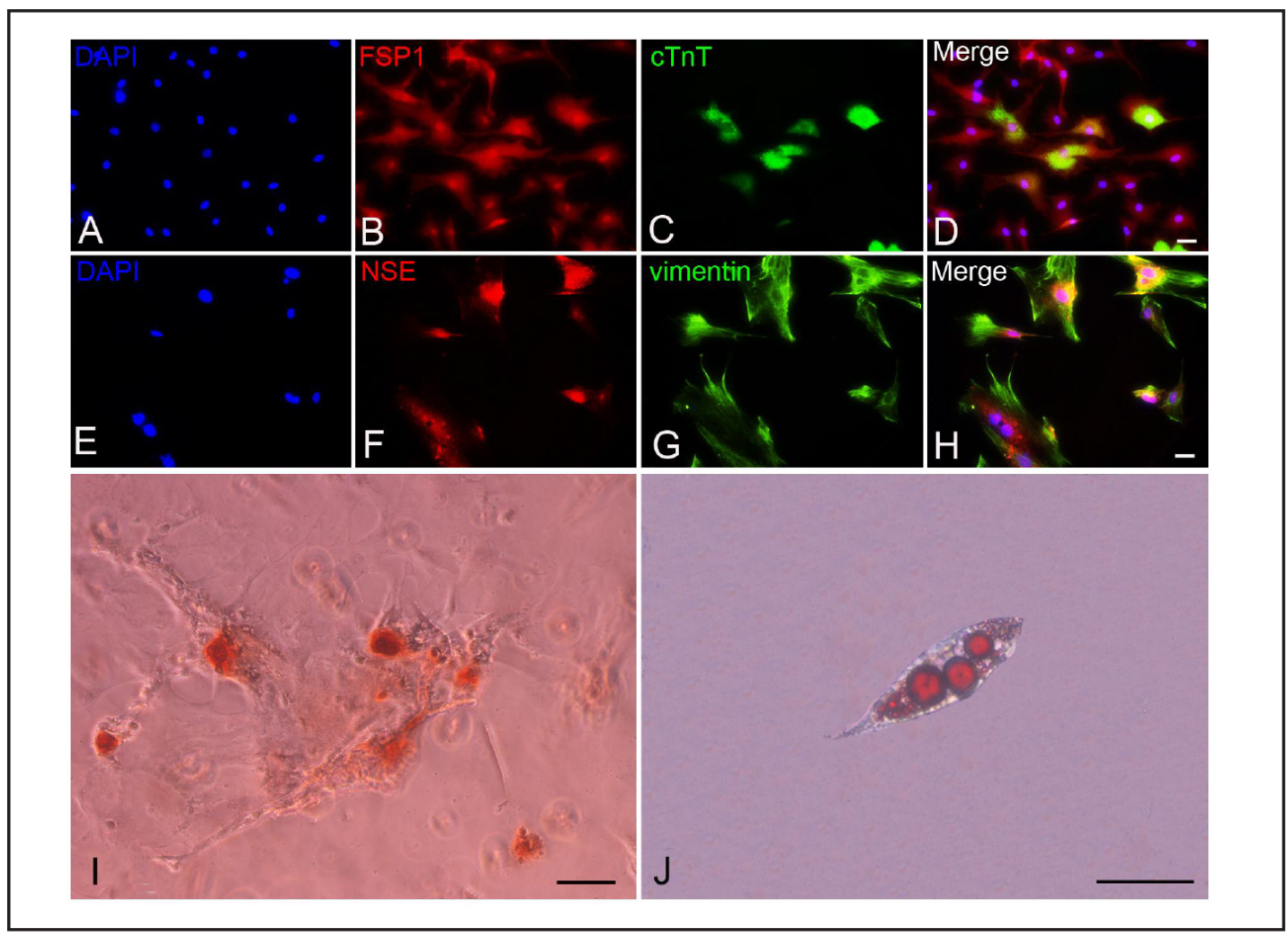

Fig. 8. Differentiation detection of fibroblasts cultured in vitro. (A-D) cardiogenic differentiation of fibroblasts after $30 \mathrm{~d}$ culture following exposure to $10 \mu \mathrm{M}$ 5-Aza for 24 hours. (A) the nuclear stained by DAPI; (B) FSP1 immunostaining. (C) CTnT immunostaining. (D) the merged image of the A-C. (E) the nuclear stained by DAPI; (F) NSE immunostaining. (G) vimentin immunostaining. (H) the merged image of the E-G. (I) Alizarin red staining, Osteogenic differentiation following exposure to osteogenic medium for $21 \mathrm{~d}$. (J) Oil Red 0 staining, adipogenic differentiation following exposure to adipogenic medium for $10 \mathrm{~d}$. Scale bar $=30 \mu \mathrm{m}$.

Fibroblasts were induced by osteogenic induction medium on 3rd, and they turned to round, oval and reniform, their volume significantly increased. Partial induced cells aggregated into colony-like with particulate matter floating on 10th day (data not shown). After $21 \mathrm{~d}$, there were many red calcium salt depositions by Alizarin red staining (Fig. 8I).

Induced fibroblasts began to become oval and shuttle shaped after being cultured by adipogenic induction medium on 2nd day and the number of cells reduced sharply, cellular processes shrinked and bigger cytoplasms. Then the cell bodies were full and nuclei were smaller after $7 \mathrm{~d}$. At the time of 10 th day, many small and round empty bubble-like structures were located in lager cytoplasms with good diopter. After $14 \mathrm{~d}$, some small and round empty bubble-like structure gradually fused into variable bigger bubbles and were detected as multilocular fat in induced fibroblasts by Oil Red 0 staining (Fig. 8J).

Cultured fibroblasts from six organs were induced into adipogenic, osteogenic, myocardial and neural differentiation respectively in vitro, which had demonstrated that fibroblasts from different organs had multipotential differentiation although there were differences in differentiation ability. Fibroblasts isolated from skin had the most advantage to differentiate into cardiomyocytes, osteocytes, adipocytes and neurons than those from other five organs (Fig. 9). Splenic fibroblasts especially had the most poor differentiation ability except for osteogenic differentiation, as there was significant difference $(P<0.01)$. Fibroblasts derived from heart, skin, liver and kidney were detected for adipogenic differentiation which accounted for 26.09-39.03\%. Fibroblasts from skin, liver, kidney and spleen had better differentiation ability in neurons. Comparing with fibroblasts from kidney, fibroblasts from 


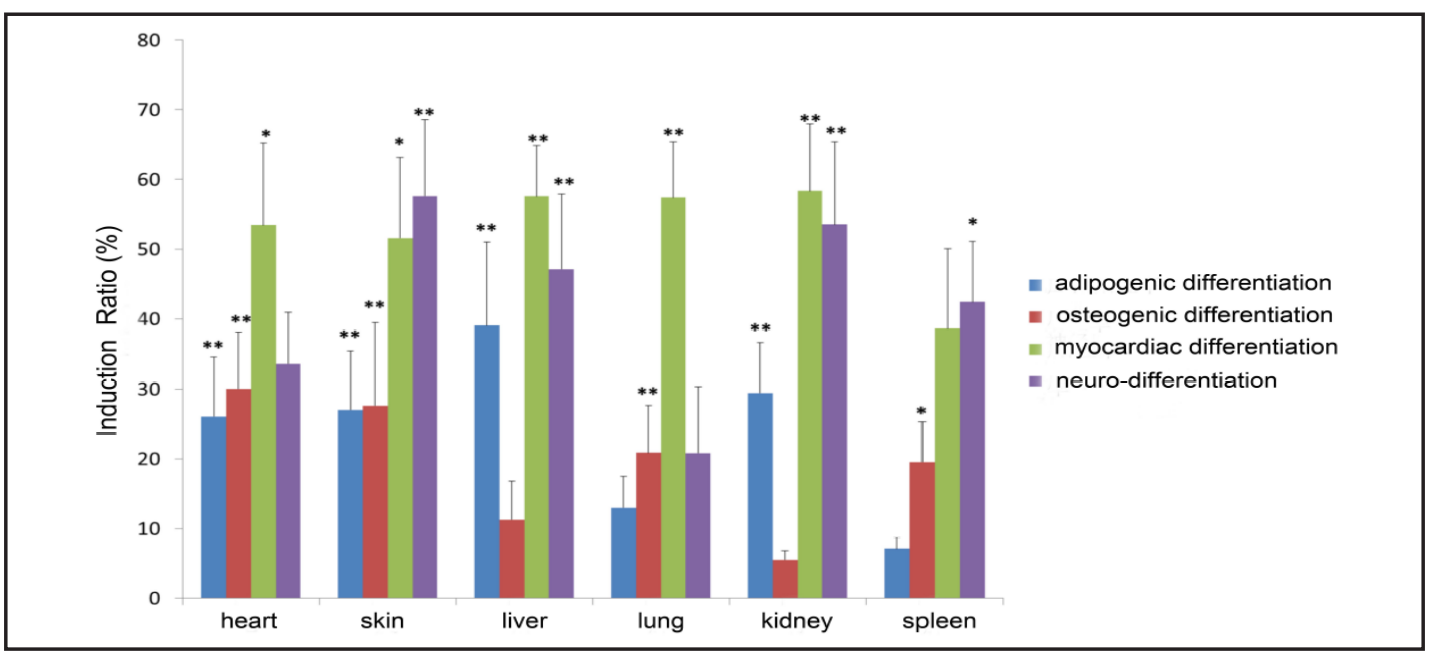

Fig. 9. Multi-directional differentiation quantitative analysis of fibroblasts from six organs. Adipogenic differentiation: fibroblasts from heart, skin, liver and kidney had significant difference comparing with in spleen $\left({ }^{* *} P<0.01\right)$. Osteogenic differentiation: comparing with in kidney, fibroblasts in spleen had difference $\left({ }^{*} P<0.5\right)$, while fibroblasts in heart, skin and lung had more significant advantages $(* * P<0.01)$. Myocardial differentiation: comparing with in spleen, fibroblasts in heart and skin had difference $\left({ }^{*} P<0.5\right)$, while fibroblasts in liver, lung and kidney had more significant advantages ( ${ }^{* *} P<0.01$ ). Differentiation ability in neurons: comparing with in lung, fibroblasts in lung and spleen had difference $\left({ }^{*} P<0.5\right)$, while fibroblasts in skin, liver and kidney had significant advantages $(* * P<0.01)$.

heart, skin and lung had more significant advantages in osteogenic differentiation $(P<0.01)$, while the differentiation ability of fibroblasts from spleen was also significantly different $(P<0.05)$. The percentage of myocardial differentiation was much higher than that of other three differentiation directions. The different myocardial differentiation ability was: fibroblasts from kidney>liver=lung $>$ heart $>$ skin $>$ spleen. Compared with fibroblasts from spleen, fibroblasts from kidney, liver and lung had significant advantage $(P<0.01)$, while fibroblasts from heart and skin were also different $(P<0.05)$.

\section{Discussion}

Fibroblasts synthesized the extracellular matrix (ECM) and collagen and played a critical role in development, the process of organ fibrosis, wound healing and angiogenesis, abundantly distributed throughout connective tissues in the organisms [12]. They were morphologically heterogeneous with diverse appearances depending on their location and activity, which showed spindle, triangle, polygon, irregular and flat star shape. Fibroblasts could also express various cytokines receptors, such as platelet derived growth factor (PDGF) and transforming growth factor $\beta 1$ (TGF- $\beta 1$ ) which would stimulate fibroblasts to proliferated rapidly and migrate into injured site once tissue damage, leading to functional impairment and scar formation [13, 14]. At present, being lack of specific markers for fibroblasts, further researches were greatly restricted.

Vimentin was a type III intermediate filament protein that was expressed in fibroblasts, endothelial cells and neurons. Given the characteristic cyto-morphological differences between these cells, anti-vimentin had been a suitable tool for reliable identification of fibroblasts. Discoidin domain receptor 2 (DDR2) was detected in cardiac fibroblasts in rodent animals $[15,16]$. DDR2 expressed also in leukocytes and tumor cells, but not expressed in cardiomyocytes, endothelial cells and smooth muscle cells [17]. In addition, Fibroblastspecific protein 1 (FSP1) was also labeled with fibroblasts [18], although FSP1 was also detected in lymphocytes and tumor cells [19]. In this study, common molecular markers 


\section{Cellular Physiology Cell Physiol Biochem 2016;39:157-171 \\ \begin{tabular}{l|l} 
and Biochemistry Published online: June 20, 2016 & $\begin{array}{l}\text { (c) } 2016 \text { The Author(s). Published by S. Karger AG, Basel } \\
\text { www.karger.com/cpb }\end{array}$
\end{tabular} \\ Chang et al.: Heterogeneity in Fibroblasts from Different Organs}

for fibroblasts were expressed differently in different organs. DDR2 was strongly expressed in fibroblasts in the heart, partly expressed in the skin, liver and spleen. Interestingly, no expression of DDR2 was detected in liver and kidney. However, vimentin and FSP1 were detected and were consistent expression in fibroblasts from skin, liver, kidney, spleen and lung. So combined expression of vimentin and FSP1 could be as effective molecular markers for fibroblast in six organs.

More recently, several reports demonstrated that fibroblasts had some heterogeneity in phenotypes and transcription patterns from different organs even though cultured in vitro [20]. Burstei et al. reported there was different morphology although with consistent gene expression [21]. Driskell et al. persisted in fibroblasts from skin were arised from two lineages [22]. One formed the upper dermis and regulated hair growth and arrector pili muscle, and the other formed the lower dermis and synthesized the bulk of the fibrillar ECM, and the preadipocytes and adipocytes of the hypodermis. The proliferation of fibroblasts from pulmonary fibrosis was obviously higher than normal control group [23]. Fibroblasts from lung had smaller cell and faster proliferation rate compared with skin fibroblasts cultured in vitro, which also supported that fibroblasts from different organs had various biological characteristics [24].

It was widely believed that mesenchymal stem cells (MSCs) were multipotential fibroblast-like cells with CD73+, CD105+, CD14-, CD34- and CD45- and plastic adherent [2527]. Recent studies had revealed that fibroblasts shared much more in common than previously recognized. Mouse embryonic fibroblasts (MEF) expressed Sca-1+, CD73+, CD105+, CD29+, CD44+, CD106+, CD11b- and CD45- [28]. Cultured fibroblasts had the positive expression in Oct-4 [29]. Dermal fibroblasts derived from rodent and human had also mesenchymal stem cell characters, such as same surface antigen and differentiation potential [30]. Our previous studies had proved that nanog was positive for cardiac fibroblasts in the infarction border zone of $1 \mathrm{~W}$ acute myocardial infarction (AMI) model [31,32]. However, it is not clear whether nanog expresses in normal organs, such as heart, lung, liver, spleen, kidney and skin. Moreover, there is still lack of comparative studies in fibroblasts on the differences in the expression of molecular markers and differentiation capacity from different organs. Therefore, cultured fibroblasts from heart, liver, lung, spleen, kidney and skin were detected for nanog, c-kit and sca-1 in gene and protein level in this study. Fibroblasts from six organs were further induced into cardiomyocytes, osteocytes, adipocytes and neurons by directed induction medium respectively.

Nanog is an important transcript factor which keeps embryonic stem cells (ESCs) selfrenewal and their pluripotent [33]. c-kit, also named stem cell growth factor receptor (SCFR), is a molecule marker of stem cells and plays an important role in cell differentiation and proliferation [34]. Bearzi et al. described c-kit ${ }^{+}$cluster in cardiac stem cell niche and confirmed endogenous cardiac stem cells(CSCs), playing an important role in myocardial regeneration and repair [35]. Stem cell antigen 1(sca-1) is another stem cell surface marker, which plays an important role in regulation of hematopoietic stem cell self-renewal, differentiation and c-kit expression [36]. Sca-1 ${ }^{+}$/CD31- CSCs can realize effective repair in myocardial infarction model [37]. Sca- $1^{+}$ESCs have strong self-regeneration and differentiation ability [38].

There are heterogeneous in c-kit and sca- 1 expression in fibroblasts from different organs, no matter in gene level or in protein expression, while there were no significant difference in nanog expression in fibroblasts from six organs $(P>0.05)$. Sca-1 expression in fibroblast from heart, liver and spleen were more than that in fibroblasts from other three organs. And c-kit expression in fibroblasts from heart was more popular than that in other five organs $(P<0.01)$. The results demonstrated that their heterogeneous phenotypes in different organs responded to the respective functions in microenviroment. Fibroblasts from heart and liver have also higher expression for stem cell markers except for fibroblasts from skin, which shows similarities in phenotypes of mesenchymal stem cells among three organs.

Chen et al. reported nestin $/$ vimentin $^{+}$dermal fibroblasts could differentiate into adipocytes, osteocytes and chondrocytes without inducer [39]. Jeney considered fibroblasts as preadipocytes due to their ability of spontaneously transferring into adipocytes [40]. 


\section{Cellular Physiology Cell Physiol Biochem 2016;39:157-171 \begin{tabular}{ll|l} 
DOI: 10.1159/000445613 & O 2016 The Author(s). Published by S. Karger AG, Basel \\
www.karger.com/cpb
\end{tabular} \\ Chang et al.: Heterogeneity in Fibroblasts from Different Organs}

Mizuno cultivated skin fibroblasts into three-dimensional collagen sponge scaffold and detected chondrocytes [41]. In this study, fibroblasts from heart, liver, spleen, lung, kidney and skin were induced into adipocytes, osteocytes, neurons and myocytes respectively, which demonstrated fibroblasts from six organs, had all multi differentiation potential in spite of their different differentiation ability. Skin fibroblasts had strong multiple differentiation potential, and the less strong one was cardiac fibroblasts. And fibroblasts in liver and kidney had advantage in myocardial differentiation, but fibroblasts in spleen only had advantage in osteogenic differentiation. And the rest had the lowest differentiation ability. Therefore, 1only partial fibroblasts from six organs in neonatal rats can differentiate into adipocytes, osteocytes, neurons and myocytes, which shows different populations in fibroblasts; 2induced adipocytes are proved to be multilocular adipocytes and maybe belong to brown adipose.

In conclusion, common molecular markers DDR2, vimentin and FSP1, stem cell markers nanog, c-kit and sca-1, differentiated advantage were systematically compared in cultured fibroblast from heart, liver, spleen, lung, kidney and skin in neonatal rats. These results provide the direct evidence that fibroblasts from different organs are heterogeneous in mesenchymal stem cell characters and fibroblasts in skin, liver and heart have stronger differential potential. It also put forward new issues to select the optimal seed cells for organ repair in situ and dominant population in fibroblasts, which is our further important topic to explore.

\section{Acknowledgements}

We thank all members of our lab for sharing reagents and advice. This work was supported by the National Natural Science Foundation of China (No. 81570268) and the Foundation and Advanced Research Plan of Henan province (No. 162300410106).

\section{Disclosure Statement}

The authors confirmed that there are no conflicts of interest.

\section{References}

1 Tsuj T, Kawada Y, Kai-Murozono M, Komatsu S, Han SA, Takeuchi K, Mizushima H, Miyazaki K, Irimura T: Regulation of melanoma cell migration and invasion by laminin-5 and alpha3beta1 integrin (VLA-3). Clin Exp Metastasis 2002;19:127-134.

2 Wang Z, Leisner TM, Parise LV: Platelet alpha2beta1 integrin activation: contribution of ligand internalization and the alpha2-cytoplasmic domain. Blood 2003;102:1307-1315.

3 Eyre DR: Collagen: molecular diversity in the body's protein scaffold. Science 1980;207:1315-1322.

4 Johansson N, Ahonen M, Kähäri VM: Matrix metalloproteinases in tumor invasion. Cell Mol Life Sci 2000;57:5-15.

5 Leppert D, Lindberg RL, Kappos L, Leib SL: Matrix metalloproteinases: multifunctional effectors of inflammation in multiple sclerosis and bacterial meningitis. Brain Res Brain Res Rev 2001;36:249-257.

6 Humphries MJ, McEwan PA, Barton SJ, Buckley PA, Bella J, Mould AP: Integrin structure: heady advances in ligand binding, but activation still makes the knees wobble. Trends Biochem Sci 2003;28:313-320.

7 Crigler L, Kazhanie A, Yoon TJ, Zakhari J, Anders J, Taylor B, Virador VM: Isolation of a mesenchymal cell population from murine dermis that contains progenitors of multiple cell lineages. FASEB J 2007;21:20502063.

8 Haniffa MA, Collin MP, Buckley CD, Dazzi F: Mesenchymal stem cells: the fibroblasts' new clothes? Haematologica 2009;94:258-263. 


\section{Cellular Physiology Cell Physiol Biochem 2016;39:157-171 \begin{tabular}{cl|l} 
DOI: 10.1159/000445613 & $\begin{array}{l}\text { O 2016 The Author(s). Published by S. Karger AG, Basel } \\
\text { www.karger.com/cpb }\end{array}$
\end{tabular} \\ Chang et al.: Heterogeneity in Fibroblasts from Different Organs}

9 Junker JP, Sommar P, Skog M, Johnson H, Kratz G: Adipogenic, chondrogenic and osteogenic differentiation of clonally derived human dermal fibroblasts. Cells Tissues Organs 2010;191:105-118.

10 Lekic PC, Pender N, McCulloch CA: Is fibroblast heterogeneity relevant to the health, diseases, and treatments of periodontal tissues? Crit Rev Oral Biol Med 1997;8:253-268.

11 Snider P, Standley KN, Wang J, Azhar M, Doetschman T, Conway SJ: Origin of cardiac fibroblasts and the role of periostin. Circ Res 2009;105:934-947.

12 Darby IA, Hewitson TD: Fibroblast differentiation in wound healing and fibrosis. Int Rev Cytol 2007;257:143-179.

13 Noronha IL, Niemir Z, Stein H, Waldherr R: Cytokines and growth factors in renal disease. Nephrol Dial Transplant 1995;10:775-786.

14 Alpers CE, Hudkins KL, Floege J, Johnson RJ: Human renal cortical interstitial cells with some features of smooth muscle cells participate in tubulointerstitialand crescentic glomerular injury. J Am Soc Nephrol 1994;5: 201-209.

15 Chin GS, Lee S, Hsu M, Liu W, Kim WJ, Levinson H, Longaker MT: Discoidin domain receptors and their ligand, collagen, are temporally regulated in fetal rat fibroblasts in vitro. Plast Reconstr Surg 2001;107:769-776.

16 Camelliti P, Borg TK, Kohl P: Structural and functional characterisation of cardiac fibroblasts. Cardiovasc Res 2005;65:40-51.

17 Goldsmith EC, Hoffman A, Morales MO, Potts JD, Price RL, McFadden A, Rice M, Borg TK: Organization of fibroblasts in the heart. Dev Dyn 2004;230:787-794.

18 Strutz F, Okada H, Lo CW, Danoff T, Carone RL, Tomaszewski JE, Neilson EG: Identification and characterization of a fibroblast marker: FSP1. J Cell Biol 1995;130:393-405.

19 Mazzucchelli L: Protein S100A4: too long overlooked by pathologists? Am J Pathol 2002;160:7-13.

20 Chang HY, Chi JT, Dudoit S, Bondre C, van de Rijn D, Brown PO: Diversity, topographic differentiation, and positional memory in human fibroblasts. Proc Natl Acad Sci USA 2002;99:12877-12882.

21 Burstein B, Libby E, Calderone A, Nattel S: Differential behaviors of atrial versus ventricular fibroblasts. Circulation 2008;117:1630-1641.

22 Driskell RR, Lichtenberger BM, Hoste E, Kretzschmar K, Simons BD, Charalambous M, Ferron SR, Herault Y, Pavlovic G, Ferguson-Smith AC, Watt FM: Distinct fibroblast lineages determine dermal architecture in skin development and repair. Nature 2013;504:277-281.

23 Jordana M, Schulman J, McSharry C, Irving LB, Newhouse MT, Jordana G, Gauldie J: Heterogeneous proliferative characteristics of human adult lung fibroblast lines and clonally derived fibroblasts from control and fibrotic tissue. Am Rev Respir Dis 1988;137:579-584.

24 Schneider EL, Mitsui Y, Au KS, Shorr SS: Tissue-specific differences in cultured human diploid fibroblasts. Exp Cell Res 1977;108:1-6.

25 Hematti P: Mesenchymal stromal cells and fibroblasts: a case of mistaken identity? Cytotherapy 2012;14:516-521.

26 Dominici M, Le Blanc K, Mueller I, Slaper-Cortenbach I, Marini F, Krause D, Deans R, Keating A, Prockop Dj, Horwitz E: Minimal criteria for defining multipotent mesenchymal stromal cells. The International Society for Cellular Therapy position statement. Cytotherapy 2006;8:315-317.

27 Choi JS, Lee BJ, Park HY, Song JS, Shin SC, Lee JC, Wang SG, Jung JS: Effects of donor age, long-term passage culture, and cryopreservation on tonsil-derived mesenchymal stem cells. Cell Physiol Biochem 2015;36:8599.

28 Saeed H, Taipaleenmaki H, Aldahmash AM, Abdallah BM, Kassem M: Mouse embryonic fibroblasts (MEF) exhibit a similar but not identical phenotype to bone marrow stromal stem cells (BMSC). Stem Cell Rev 2012;8:318-328.

29 Szabo E, Rampalli S, Risueňo RM, Schnerch A, Mitchell R, Fiebig-Comyn A, Levadoux-Martin M, Bhatia M: Direct conversion of human fibroblasts to multilineage blood progenitors. Nature 2010;468:521-526.

30 Lorenz K, Sicker M, Schmelzer E, Rupf T, Salvetter J, Schulz-Siegmund M, Bader A: Multilineage differentiation potential of human dermal skin-derived fibroblasts. Exp Dermatol 2008;17:925-932.

31 Luo H, Li Q, Pramanik J, Luo J, Guo Z: Nanog expression in heart tissues induced by acute myocardial infarction. Histol Histopathol 2014;29:1287-1293.

32 Chang Y, Li H, Guo Z: Mesenchymal stem cell-like properties in fibroblasts. Cell Physiol Biochem 2014;34:703-714 


\section{Cellular Physiology Cell Physiol Biochem 2016;39:157-171 \begin{tabular}{l|l} 
DOI: 10.1159/000445613 & $\begin{array}{l}\text { O 2016 The Author(s). Published by S. Karger AG, Basel } \\
\text { www.karger.com/cpb }\end{array}$
\end{tabular} \\ Chang et al.: Heterogeneity in Fibroblasts from Different Organs}

33 Chambers I, Colby D, Robertson M, Nichols J, Lee S, Tweedie S, Smith A: Functional expression cloning of Nanog, a pluripotency sustaining factor in embryonic stem cells. Cell 2003;113:643-655.

34 Edling CE, Hallberg B: c-Kit-a hematopoietic cell essential receptor tyrosine kinase. Int. J. Biochem. Cell Biol 2007;39:1995-1998.

35 Bearzi C, Rota M, Hosoda T, Tillmanns J, Nascimbene A, De Angelis A, Yasuzawa-Amano S, Trofimova I, Siggins RW, Lecapitaine N, Cascapera S, Beltrami AP, D’Alessandro DA, Zias E, Quaini F, Urbanek K, Michler RE, Bolli R, Kajstura J, Leri A, Anversa P: Human cardiac stem cells. Proc Natl Acad Sci USA 2007;104:14068-14073.

36 Wang X, Hu Q, Nakamura Y, Lee J, Zhang G, From AH, Zhang J: The role of the sca-1+/CD31- cardiac progenitor cell population in postinfarction left ventricular remodeling. Stem Cells 2006;24:1779-1788.

37 Bradfute SB, Graubert TA, Goodell MA: Roles of Sca-1 in hematopoietic stem/progenitor cell function. Exp Hematol2005; 33:836-843.

38 Montemurro F, Ueno NT, Rondón G, Aglietta M, Champlin RE: High-dose chemotherapy with hematopoietic stem-cell transplantation for breast cancer: current status, future trends. Clin Breast Cancer 2000;1:197209.

39 Chen FG, Zhang WJ, Bi D, Liu W, Wei X, Chen FF, Zhu L, Cui L, Cao Y: Clonal analysis of nestin(-) vimentin(+) multipotent fibroblasts isolated from human dermis. J Cell Sci 2007;120:2875-2883.

40 Jeney F, Bazsó-Dombi E, Oravecz K, Szabó J, Nagy IZ: Cytochemical studies on the fibroblast-preadipocyte relationships in cultured fibroblast cell lines. Acta Histochem 2000;102:381-389.

41 Mizuno S, Glowacki J: Low oxygen tension enhances chondroinduction by demineralized bone matrix in human dermal fibroblasts in vitro. Cells Tissues Organs 2005;180:151-158. 